\title{
STUDY OF CORONA PULSES IN AIR-SOLID INTERFACE
}

\author{
R. S. Nema \\ Department of High Voltage Engineering \\ Indian Institute of Science \\ Bangalore - 560 012, INDIA \\ nema@hve.iisc.ernet.in
}

\author{
Fathima Zahra \\ Faculty of Engineering and Applied Science \\ Memorial University of Newfoundland \\ St. John's, NF, CANADA A1B 3X5 \\ fathima@engr.mun.ca
}

\begin{abstract}
In most of the engineering applications, air is used in conjunction with solid insulation. The interface forms a weak point for the occurrence of partial discharges (PD). Therefore it becomes essential to know the behaviour of the PD in such a system. The work presented in the paper is a study of the PD corona pulse shape in air-solid interface under varying conditions of gap and pressure. Some results on the pulse height distributions of the PD in air-solid interface are also highlighted.
\end{abstract}

\section{Introduction}

Partial discharges are considered to be the major reason for the aging and eventual failure of electrical insulation. Hence one of the aspect of today's PD analysis is the assessment of the actual insulation conditions by using PD as an indicator of electrical insulation degradation. Thus the influence and effect of PD on a solid insulation are still under intense investigation. It is quite common that air adjacent to solid dielectric is under electric stress. Examples of the air-solid type of interface include the leads from the high voltage winding of a transformer to its bushings, passing through air or the end portion of a high voltage stator coil in a synchronous generator which is partly insulated with a solid dielectric and partly by a definite thickness of air. With alternating voltage, the space charges tend to migrate during each half cycle, so that the repetition rate is a function of frequency. It is well established that the accumulation of space charge produced during the discharge activity acts to reduce the local electric field strength to a level insufficient to sustain the discharge [1].

Discharge parameters determining the development of a discharge are contained in the pulse shape. To obtain information about the discharge induced degradation process, it is necessary to record the discharge with a bandwidth large enough to retain the original pulse shape of the signal. The method of pulse height analysis is applied to analyze PD data in terms of the number, phase of occurrence as well as the magnitude of the pulse.

Basic parameters of the PD signals can be classified into 3 quantities; the phase angle $\phi$, pulse charge $q$ and the pulse repetition rate $n$.PD phase angle is defined as the ac applied voltage phase angle at which PD takes place. The discharge magnitude represents the peak value of the single discharge pulse.

\section{Experimental Setup}

The block diagram of the experimental setup is shown in Fig. 1. To extract the undistorted information of the pulse, a sub-divided measuring electrode system is used. This provides a closed, high-frequency path with low stray capacitance [2]. The sub-divided bottom electrode has a measuring disc of $20 \mathrm{~mm}$ diameter and the ground electrode is of $60 \mathrm{~mm}$ outer diameter, with an annular spacing of 1 $\mathrm{mm}$ between them. The top electrode is a needle tip of diameter $0.31 \mathrm{~mm}$

A stainless steel chamber is used as a test cell and is covered with a PMMA (Poly methyl methacrylate) lid. A resin bonded epoxy bushing is fitted to this lid for applying high voltages to the top electrode. The cell has a port that is connected to a vacuum pump system for creating low pressures inside the chamber. A Wilson-seal arrangement is attached to the bottom electrode, making it possible to vary the gap between the electrodes with the help of a micrometer screw gauge.

The measuring resistor consists of six $300 \Omega$ metal film resistors connected between the stem of the measuring disc and guard ring of the bottom electrode. The output across the measuring resistor is taken outside the chamber using a small low voltage PTFE (Poly tetra fluoroethylene) bushing. The voltage is applied from a $50 \mathrm{kV}, 2 \mathrm{kVA}$ transformer which is discharge free upto $22 \mathrm{kV}$. The test voltage is applied to the specimen through a bandpass filter which isolates the noise of the transformer from the measuring circuit. A $1 \mathrm{M} \Omega$ damping resistor is connected close to the top electrode and provides protection in case of a complete breakdown and prevents the reflections of high voltage frequency currents on the high voltage lead. The measuring resistance is $25 \Omega$, formed by two terminating $50 \Omega$ resistors at each end of a coaxial cable leading to a TDS $684 \mathrm{~A}$ 


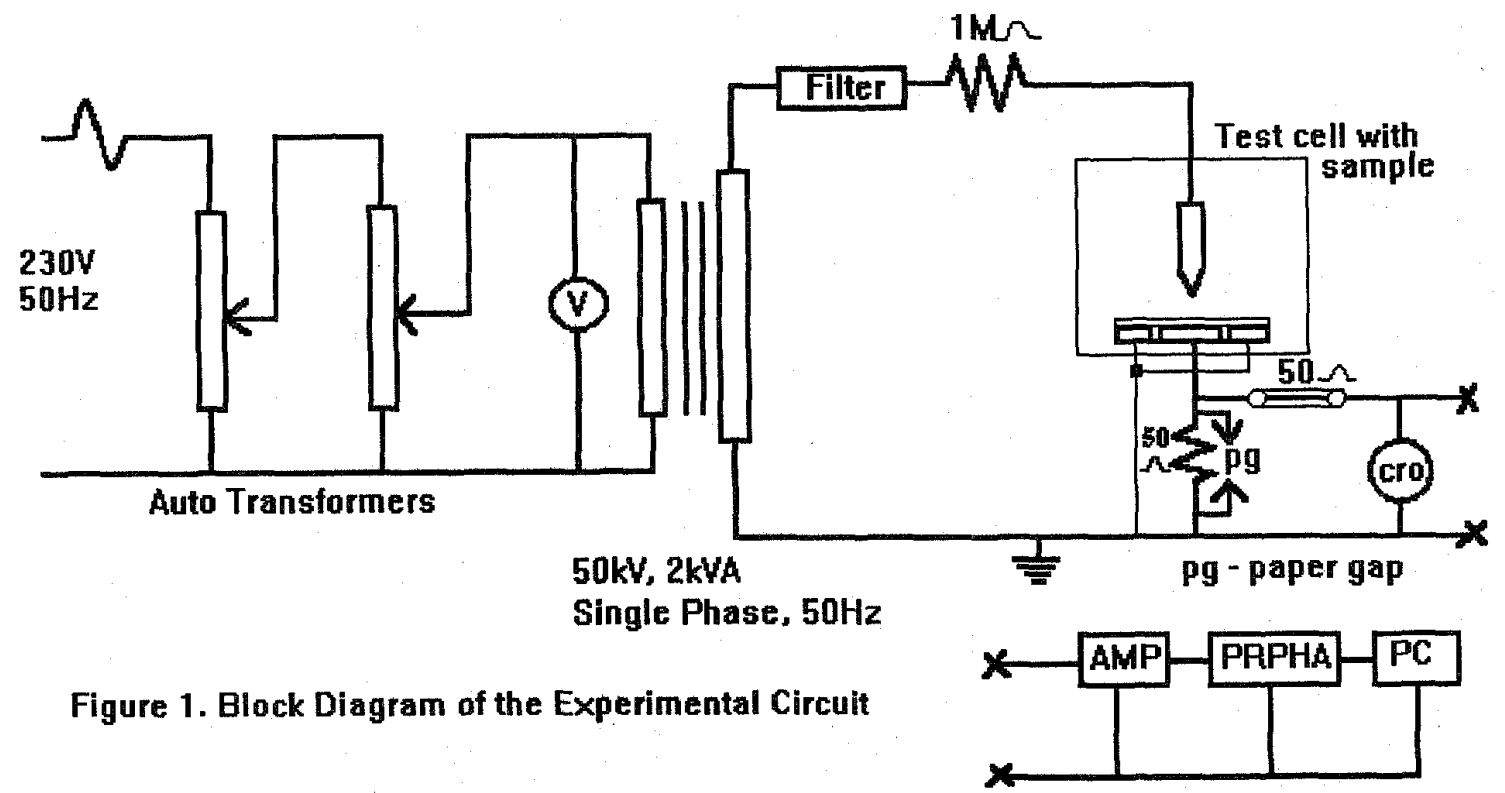

Tektronix digital oscilloscope having $1 \mathrm{GHz}$ bandwidth and a sampling rate of 5 Giga samples/sec.

The experiments are carried out at atmospheric pressure of $680 \mathrm{~mm} \mathrm{Hg}$ (local atmospheric) and $100 \mathrm{~mm} \mathrm{Hg}$ (low pressure). While conducting experiments under low pressure, care is taken to seal the test chamber tightly. The pressure inside the chamber is measured by an open ended mercury manometer with an accuracy of $1 \mathrm{~mm} \mathrm{Hg}$. PMMA is used as the sample, having a thickness of $1.5 \mathrm{~mm}$ and diameter of $60 \mathrm{~mm}$. Gap distances from $2.5 \mathrm{~mm}$ to $5 \mathrm{~mm}$ in steps of $0.5 \mathrm{~mm}$ are chosen for conducting the experiments. To obtain information on the pulse height distribution, the discharge pulse is fed to an amplifier (AMP) and a phase resolved pulse height analyzer (PRPHA). The resolution time is $10 \mu \mathrm{s}$ and the maximum repetition rate is $100 \mathrm{k}$ pulses/sec.

\subsection{Measurements}

The partial discharge corona inception voltage (PDCIV) is taken as that minimum voltage at which discharge pulses appeared at every negative half cycle. At any gap, the voltage is applied and at the appearance of the first pulse, the output voltage is noted. This procedure is repeated 10 times and the average is taken as the PDCIV. The same is repeated for different gaps and the corresponding values of the PDCIV are noted. The above procedure is repeated at different pressures.

The voltage pulses across the measuring resistor are captured by the TDS $684 \mathrm{~A}$ digital oscilloscope using it in a single shot mode. The rise time, fall time, magnitude and area of each pulse at the PDCIV are noted. For each measurement, 10 recordings are obtained. A time interval of 5 minutes is selected between the recordings. At different gaps, the same procedure is repeated.

For obtaining the pulse height distributions, the data acquisition time is taken as $100 \mathrm{~ms}$. One cycle of data from each set is considered for the analysis.

\section{Experimental Results}

\subsection{Discharge Pulses at Atmospheric Pressure}

Figure 2 represents the pulse shape obtained for a total gap of $5 \mathrm{~mm}$ including the dielectric at $680 \mathrm{~mm} \mathrm{Hg}$. As seen from Table 1, the rise time of the pulse almost remains constant, but the fall time increases. Minimum rise time in some cases was found to be 800 ps. The magnitude of the pulse is found to decrease slightly with the increase in the gap, presumably due to higher gap impedance.

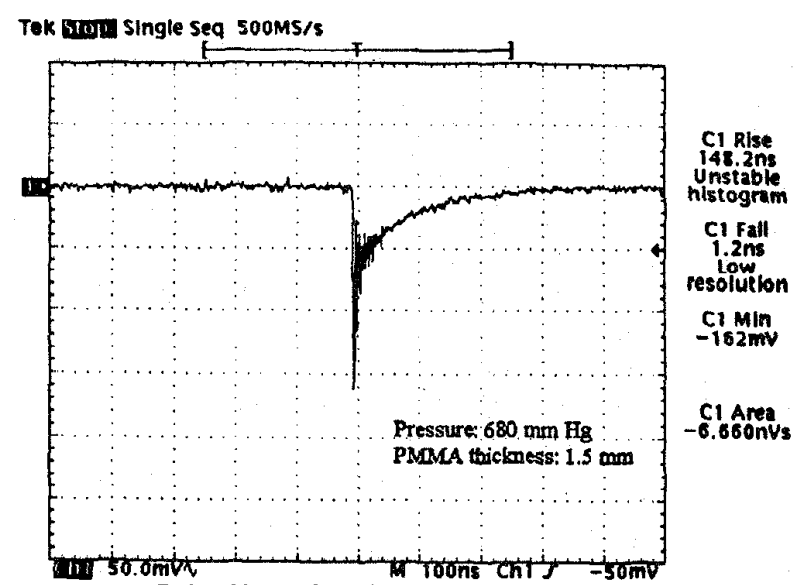

Fig 2. Pulse Shape for Air and PMMA gap of $5 \mathrm{~mm}$

A more detailed work has been carried out at over voltages also [3]. It was found that the rise time, fall time, magnitude 
as well as area of the pulse differs from those pulses obtained at inception voltage. When the medium was air gap alone, it was observed that the rise time of the pulses slightly increased. Also an interesting result is that the pulse shapes obtained for a pressure of $200 \mathrm{~mm} \mathrm{Hg}$ were almost of the same nature as that of the pulses obtained at $680 \mathrm{~mm} \mathrm{Hg}$ [3].

\subsection{Discharge Pulses at Low Pressure}

A typical discharge pulse obtained for a total gap of $5 \mathrm{~mm}$ at $100 \mathrm{~mm} \mathrm{Hg}$ is shown in figure 3. As seen from table 2, the rise time does not change much with increasing gap. The fall time and the area however increases, whereas the magnitude is found to decrease. It is observed that the rise time of the pulse is comparatively high when compared with the pulses obtained at atmospheric pressure. As pressure decreases, the fall time increases.

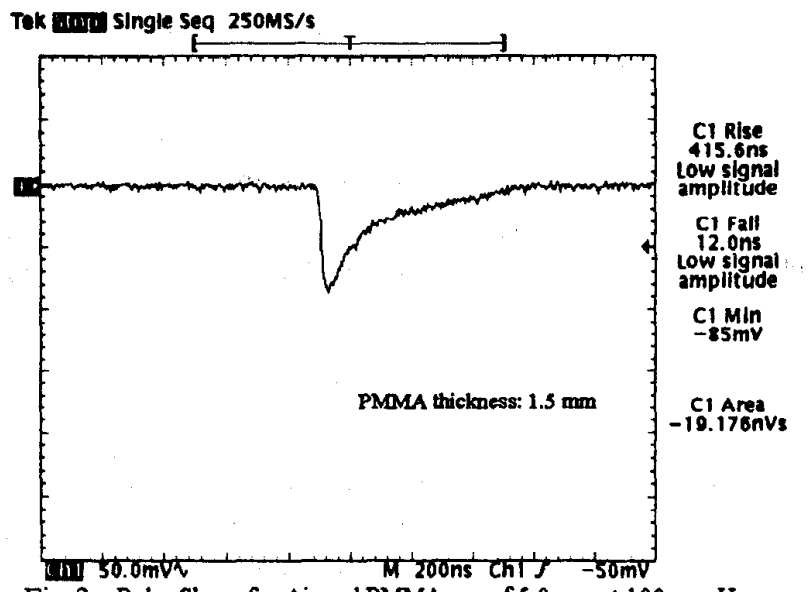

Fig 3. Pulse Shape for Air and PMMA gap of $5.0 \mathrm{~mm}$ at $100 \mathrm{~mm} \mathrm{Hg}$

At low pressure in presence of a dielectric, it is noted that the pulses are by nature due to streamer discharge [4]. This was not the case for pulses in presence of air gap alone at low pressures [3]. It is found that the discharge is a Townsend type at reduced pressures, when the medium is air gap alone.

\subsection{Pulse Height Distributions}

The $\phi-n-q$ (phase-number-charge) distributions for a total gap of $5 \mathrm{~mm}$ at $680 \mathrm{~mm} \mathrm{Hg}$ and $100 \mathrm{~mm} \mathrm{Hg}$ is shown in figures 4 and 5 respectively. The shape of distributions resembles a normal one. Positive pulses were also seen when the dielectric is present in the gap. It is seen that the number of middle order pulses are more. As such, the number of pulses are less when compared to the distributions obtained in air gap alone, since there is a build up of charge due to the dielectric

\section{Discussion}

In presence of the dielectric, the corona pulses are found to be intermittent in nature. This is because when a discharge strikes the dielectric, the charge accumulates on the surface thus reducing the electric field strength at the point electrode. This will inhibit the recurrence of the discharges and hence more intermittence [5].

The ionization and breakdown are due to streamer mechanism [4]. The process of ionization is due to multiple electron avalanches and hence fast rise times are obtained. The drift velocity of electrons can be of the order of $10^{8}$ $\mathrm{cm} / \mathrm{sec}$ and ionic drift velocity $10^{6} \mathrm{~cm} / \mathrm{sec}$ [4]. Therefore, in the gaps here, the expected rise time due to electron mobility will be of the order of a nanosecond and the fall time due to the ions will be about 100 ns. As the total gap increases, it is found that the fall time increases. This is due to the surface charge field of the dielectric which may not allow the recombination processes in the gas phase. The prescnce of these surface charges gives rise to the long fall times and helps in ion-electron recombination resulting in less charge in the pulse, thus reducing the magnitude of the pulse [1]. When a dielectric is inserted, the variation of the electric field strength is quite large compared to air gap alone. This clearly gives an indication that the charges on the dielectric and particularly at small gaps alter the field more significantly.

The $\phi-n-q$ patterns clearly indicate the change in the number of pulses, their magnitude as well as the phase of occurrence at different pressures. The results obtained show a statistical variation in phase and magnitude and these can be attributed to the memory effects [5]. The distributions were analyzed using skewness and kurtosis [6]. At atmospheric pressure, both skewness and kurtosis had positive values. At low pressure, for overvoltages, the values were negative. Thus skewness and kurtosis give an indication of the degree of insulation degradation resulting from $\mathrm{PD}$.

\section{Conclusions}

From the above results, it is seen that there is a significant variation in the pulse shape at different pressures. The fall time, magnitude as well as the area of the pulse are altered. It is observed that the pulses are intermittent in nature in the presence of a dielectric. With the dielectric, pulses of rise times of upto 800 ps have been found to exist. Hence by observing the pulse shapes, it is possible to get a knowledge of the conditions under which the discharge occurred. As can be seen from the oscillograms and the tables, even the slightest variations of the gap conditions influence the pulse shape. The $\phi-n-q$ patterns give additional information of the number and phase of occurrence. Thus the pulse shape and the $\phi-n-q$ distributions can form a pattern recognition parameter. 


\section{References}

1. R. J. Van Brunt, 'Physics and Chemistry of Partial Discharges and Corona - Recent advances and future challenges", IEEE Trans. on Electrical Insulation and Dielectric Phenomena, Vol. 1, No. 5, pp. 761-784, 1994.

2. P. H. F. Morshius, "Time Resolved Discharge Measurements", IEE Proceedings on Intl. Conf. on Partial Discharges, pp. 43-46, 1993.

3. Fathima Zahra, 'Partial Discharge Corona Pulse Characterization in Air and Air-solid Interface",
Masters Thesis, Indian Institute of Science, Bangalore, India, September 1996.

4. E. Nasser, Fundamentals of Gaseous Ionization and Plasma Electronics, Wiley Publications, 1971.

5. R. J. Van Brunt and S. V. Kulkarni, 'Method for Measuring the Stochastic Properties of Corona and Partial Discharge Pulses", Review Sci. Instrum. 60 (9), pp. 3012-3023, September 1989.

6. Bartnikas and J. P. Novak, 'On the character of different forms of Partial Discharges and their Related Terminologies", IEEE Trans. on Electrical Insulation, Vol. 28, No. 6, pp. 956-968, December 1993.

Table 1: Variation of pulse shape parameters with air and PMMA at $680 \mathrm{~mm} \mathrm{Hg}$

\begin{tabular}{|c|c|c|c|c|}
\hline Gap Length (mm) & Rise Time (ns) & Fall Time (ns) & Magnitude (mV) & Area (nVs) \\
\hline 2.5 & 1.60 & 75.67 & 185.90 & 5.816 \\
\hline 3.0 & 1.51 & 102.90 & 152.31 & 5.837 \\
\hline 3.5 & 1.43 & 118.63 & 132.33 & 5.428 \\
\hline 4.0 & 1.60 & 115.17 & 132.71 & 5.791 \\
\hline 4.5 & 1.55 & 145.20 & 145.20 & 5.448 \\
\hline 5.0 & 1.67 & 129.13 & 122.19 & 5.218 \\
\hline
\end{tabular}

Table 2: Variation of pulse shape parameters with air and PMMA at $100 \mathrm{~mm} \mathrm{Hg}$

\begin{tabular}{|c|c|c|c|c|}
\hline Gap Length (mm) & Rise Time (ns) & Fall Time (ns) & Magnitude (mV) & Area (nVs) \\
\hline 2.5 & 9.60 & 172.05 & 115.50 & 7.069 \\
\hline 3.0 & 12.80 & 176.20 & 90.50 & 7.188 \\
\hline 3.5 & 11.01 & 281.50 & 119.00 & 12.067 \\
\hline 4.0 & 13.60 & 345.30 & 97.50 & 16.078 \\
\hline 4.5 & 12.30 & 455.65 & 83.25 & 16.650 \\
\hline 5.0 & 13.20 & 497.36 & 94.00 & 20.280 \\
\hline
\end{tabular}

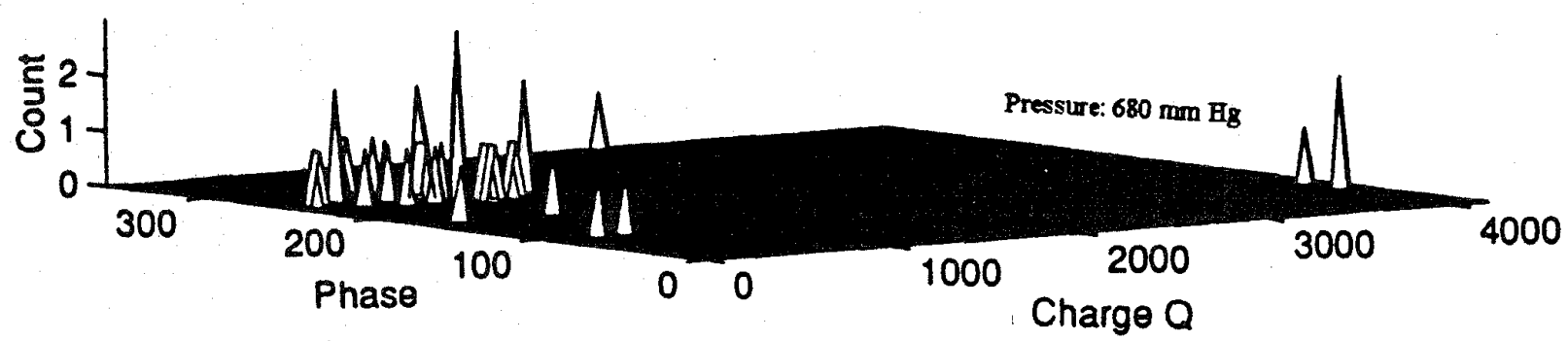

Fig 4. $p-n-q$ Distribution For Air and Dielectric Gap of $5 \mathrm{~mm}$ at $3.58 \mathrm{kV}$

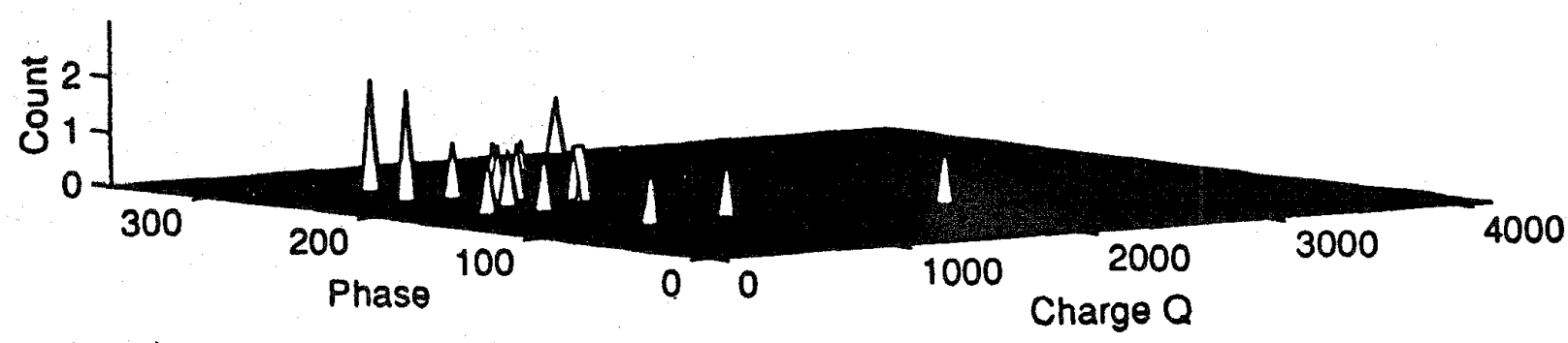

Fig 5. $q-n-q$ Distribution For Air and Dielectric Gap of $5 \mathrm{~mm}$ at $1.82 \mathrm{kV}$ and $100 \mathrm{~mm}$ of $\mathrm{Hg}$ 